\title{
MODELLING OF PULSATING DIFFUSIONAL BOUNDARY LAYERS-II A REVERSIBLE REDOX ELECTROCHEMICAL REACTION INVOLVING SOLUBLE SPECIES IN SOLUTION THEORY AND EXPERIMENTAL TEST
}

\author{
C I Elsner, C L Perdriel, S L Marchiano and A J Arvia
}

Instituto de Investigaciones Fisıcoquimicas Teoricas y Aplicadas (INIFTA) Facultad de Ciencias

Exactas, Unıversidad Nacional de La Plata Sucursal 4, Casilla de Correo 16, (1900) La Plata, Argentına

(Recelved 13 December 1988, in revised form 10 May 1989)

\begin{abstract}
A solution of the diffusion equation for a reversible redox electrochemical reaction in the presence of only one species (the reactant) and in the absence of products at the initiation of the reaction for a square wave perturbing potentıal is presented The solution furnishes the concentration profiles for reactant and product and their dependence on the time elapsed from the initiation of the halfcycle $t^{\prime}$, on the frequency of the periodic potential, on the number of cycles, $N$, and on the symmetry of the signal In addition, the dependences of the current on $t^{\prime}$ and $N$, and that of the charge density on $N$, were obtained Conclusions from the theory are experimentally verified by using the reversible $\left[\mathrm{Fe}(\mathrm{CN})_{6}\right]^{4-} /\left[\mathrm{Fe}(\mathrm{CN})_{6}\right]^{3-}$ redox couple on platınum as a test reaction
\end{abstract}

\section{INTRODUCTION}

The application of periodic perturbing potentials to electrodes in contact with electrolyte solutions produces different changes at the interphase depending on the potential limits $\left(E_{\mathrm{a}}\right.$ and $\left.E_{\mathrm{c}}\right)$, frequency $(f)$ and time-symmetry of the perturbation[1-5] For a simple electrochemical reaction involving soluble species in solution, these changes can be assigned to modifications of charging of the electrical double layer (nonfaradaic process) and variation of local concentrations of reactant and product in the vicinity of the electrode caused by the faradaic process For the sake of simplicity, one can consider as a first approach that the relative contribution of non-faradaic to faradaic processes is negligible When the faradaic process proceeds by applying a penodic perturbing potential and occurs under either diffusion or intermediate kinetics control, concentration gradients at the electrodesolution interphase are established Correspondingly, pulsating diffusion boundary layers are built up for the concentrations of reactant and product involved in both the anodic and cathodic reactions, respectively The general behaviour of each one of these layers for a fixed geometry depends on the properties of the solution, kinetic parameters of the electrochemical reaction and perturbing potential conditions Thus, for a particular electrochemical reaction subjected to a tıme-symmetric square wave perturbing potentıal, which is potential-symmetric with respect to its equilibrium potentıal, the average thıcknesses of the diffusion boundary layers for a constant number of cycles decrease as $f$ increases [5]

As far as the entire kinetics of the electrochemical reaction is concerned, the frequency dependence of the diffusion boundary layer thickness brings about various important consequences Firstly, the fact that, in principle, a frequency, $f_{t}$, should be found at which one can observe a transition in the kinetics of the reaction from a mass transport control, for $f<f_{\mathrm{t}}$, to an activation control, for $f>f_{t}$ This transition depends on the $\left[k_{\mathrm{f}} / D_{\mathrm{ox}}^{1 / 2}+k_{\mathrm{b}} / D_{\mathrm{re}}^{1 / 2}\right]$ term $[6,7]$, where $k_{\mathrm{f}}$ and $k_{\mathrm{b}}$ are the kinetic constants for the forward and backward reactions, respectively, and $D_{\text {re }}$ and $D_{\text {ox }}$ denote the diffusion coefficients of species entering the anodic and cathodic reactions, respectively Detals about the kinetic transition deserves an extensive discussion in a forthcoming publication[8] Secondly, it should be noticed that the validity of the electroneutrality condition should depend on the rate of mass transport and frequency of the periodic perturbing potential Thus, in dealing with a fast electrochemical reaction at low values of $f$, the thickness of the diffusion boundary layers become sufficiently large to fullfill the electroneutrality requirement for solving the pure diffusion problem as expressed throughout Fick's equations [7] However, a more complex situation arises at large values of $f$, te small thickness of the diffusion boundary layer and diluted electrolyte solution where an increasing departure from the above mentioned cond1tion should be expected[9]

The present work is devoted to solve the diffusion equation for a fast redox electrochemical reaction produced by means of a square wave perturbing potential in the absence of products at the initiation of the reaction The theoretical approach considers that non-faradaic processes are negligible, that the electroneutrality condition at the diffusion boundary layer is satısfied and, correspondingly, that there is no migration contribution term Furthermore, the redox electrochemical process behaves as an ideal reversible reaction, te it implies a large exchange current density value In this way the model allows to explicit the concentration profiles for reactant and product the influence of $f$ and the number of cycles, $N$, as well as the influence of the symmetry of the perturbing poten- 
tial Conclusions from the theory are experimentally verified by studying an electrochemical test reaction in a suitable cell design These results are relevant to deal with the more complex situations precedingly described

\section{SOLUTION OF THE DIFFUSION EQUATION}

Let us consider a fast electrode reaction involving soluble species in solution represented by the equation

$$
\mathrm{ox}+\mathrm{e}^{-}=\mathrm{re},
$$

which takes place under diffusion control at an inert solid semi-1nfinite plane electrode The oxidized and reduced states of the species in solution are denoted as ox and re respectively For the selected electrode geometry, the concentration profiles of ox and re at any instant, can be obtained by solving Fick's equation for each species in the $x$-direction, the latter being chosen perpendicular to the electrode surface

$$
\frac{\partial c_{\mathrm{ox}}(x, t)}{\partial t}=D_{\mathrm{ox}} \frac{\partial^{2} c_{\mathrm{ox}}(x, t)}{\partial x^{2}},
$$

and

$$
\frac{\partial c_{\mathrm{re}}(x, t)}{\partial t}=D_{\mathrm{re}} \frac{\partial^{2} c_{\mathrm{re}}(x, t)}{\partial x^{2}},
$$

where the $c_{\mathrm{ox}}$ and $c_{\mathrm{re}}$ refer to the concentrations of ox and re, respectively, and $D_{\mathrm{ox}}$ and $D_{\mathrm{re}}$, the corresponding diffusion coefficients which are assumed to be $c l^{\prime}$ independent values

\section{The general case}

Reaction (1) proceeds under a square wave perturbing potential, te successive cathodic, $E_{\mathrm{c}}$, and anodic, $E_{\mathrm{a}}$, potential steps The frequency of the perturbing potential is $f=1 / T=1 /\left(\tau_{\mathrm{a}}+\tau_{\mathrm{c}}\right)$, where $T$ is the period, and $\tau_{\mathrm{a}}$ and $\tau_{\mathrm{c}}$ are the anodic and cathodic halfperiods, respectively The values of $E_{\mathrm{a}}$ and $E_{\mathrm{c}}$ are set in such a way that $E_{\mathrm{a}}>E_{\mathrm{r}}^{\circ}$ and $E_{\mathrm{c}}<E_{\mathrm{r}}^{\mathrm{o}}$, $E_{\mathrm{r}}^{\mathrm{o}}$ denoting the standard potential of redox reaction (1)

To solve Fick's equations the following initial and boundary conditions are considered For $t=0$,

$$
\left.\begin{array}{l}
c_{\mathrm{ox}}=c^{0} \\
c_{\mathrm{re}}=0
\end{array}\right] \text {, }
$$

and for $t>0$ and $x=0$

$$
\begin{aligned}
& \frac{c_{\mathrm{ox}}(0, t)}{c_{\mathrm{re}}(0, t)}=\theta=\exp \left[\frac{z \mathrm{~F}}{\mathrm{R} T}\left(E_{\mathrm{c}}-E_{\mathrm{r}}^{0}\right)\right], \\
& \text { for odd halfcycles, } \\
& \frac{c_{\mathrm{ox}}(0, t)}{c_{\mathrm{re}}(0, t)}=\psi=\exp \left[\frac{z \mathrm{~F}}{\mathrm{R} T}\left(E_{\mathrm{a}}-E_{\mathrm{r}}^{0}\right)\right],
\end{aligned}
$$

for even halfcycles,

and

$D_{\mathrm{ox}}\left(\frac{\hat{\partial} c_{\mathrm{ox}}(x, t)}{\partial x}\right)_{x=0}+D_{\mathrm{re}}\left(\frac{\partial c_{\mathrm{re}}(x, t)}{\partial x}\right)_{x=0}=0$ where the tıme $t$, is defined as follows

$$
t=((N-1) / 2)\left(\tau_{\mathrm{a}}+\tau_{\mathrm{c}}\right)+t^{\prime}, \text { for odd values of } N
$$
and

$t=(N / 2) \tau_{\mathrm{c}}+(N / 2-1) \tau_{\mathrm{a}}+t^{\prime}$, for even values of $N$, where

$$
\begin{aligned}
& 0<t^{\prime}<\tau_{c}, \quad \text { for odd values of } N, \\
& 0<t^{\prime}<\tau_{a}, \text { for even values of } N,
\end{aligned}
$$

and $N$ represents the total number of halfcycles For $N=1$, the first cathodic halfcycle, the concentration profiles for ox and re are given by the well-known equations given elsewhere[7]

The general solution of Fick's equations for any value of $N$ for the cathodic and the anodic halfcycles respectively, are given by the following expressions For odd values of $N$

$$
\begin{aligned}
c_{\mathrm{ox}}(x, t)= & \frac{1}{1+\gamma^{1 / 2} \theta} \\
& \times\left[\gamma^{1 / 2} \theta+\sum_{k=1}^{(N+1) / 2} \operatorname{erf} \frac{x}{2 \sqrt{D_{\mathrm{ox}}} \sqrt{A+t^{\prime}}}\right. \\
& -\sum_{k=0}^{(N-2) / 2} \operatorname{erf} \frac{x}{\left.2 \sqrt{D_{\mathrm{ox}}} \sqrt{B+t^{\prime}}\right]} \\
& +\frac{1}{1+\gamma^{1 / 2} \psi}\left[\sum_{k^{*}=1}^{(N-1) / 2} \operatorname{erf} \frac{x}{2 \sqrt{D_{\mathrm{ox}}}} \sqrt{E+t^{\prime}}\right. \\
& \left.-\sum_{k=0}^{(N-2) / 2} \operatorname{erf} \frac{x}{2 \sqrt{D_{\mathrm{ox}}} \sqrt{P+t^{\prime}}}\right]
\end{aligned}
$$

and

$$
\begin{aligned}
\frac{c_{\mathrm{re}}(x, t)}{c^{0}}= & \frac{\gamma^{1 / 2}}{1+\gamma^{1 / 2} \theta}\left[1+\sum_{k=0}^{(N-2) / 2} \operatorname{erf} \frac{x}{2 \sqrt{D_{\mathrm{rc}}} \sqrt{B+t^{\prime}}}\right. \\
& \left.-\sum_{k=1}^{(N+1) / 2} \operatorname{erf} \frac{x}{2 \sqrt{D_{\mathrm{re}}} \sqrt{A+t^{\prime}}}\right]+\frac{\gamma^{1 / 2}}{1+\gamma^{1 / 2} \psi} \\
& \times\left[\sum_{k=0}^{(N-2) / 2} \operatorname{crf} \frac{x}{2 \sqrt{D_{\mathrm{re}}} \sqrt{P+t^{\prime}}}\right. \\
& \left.-\sum_{k^{*}=1}^{(N-1) / 2} \operatorname{erf} \frac{x}{2 \sqrt{D_{\mathrm{re}}} \sqrt{E+t^{\prime}}}\right]
\end{aligned}
$$

and for even values of $N$

$$
\begin{aligned}
\frac{c_{\mathrm{ox}}(x, t)}{c^{0}}= & \frac{1}{1+\gamma^{1 / 2} \theta}\left[\sum_{k=0}^{(N-2) / 2} \operatorname{erf} \frac{x}{2 \sqrt{D_{\mathrm{ox}}} \sqrt{G+t^{\prime}}}\right. \\
& \left.-\sum_{k=1}^{(N+1) / 2} \operatorname{erf} \frac{x}{2 \sqrt{D_{\mathrm{ox}}} \sqrt{A+t^{\prime}}}\right]+\frac{1}{1+\gamma^{1 / 2} \psi} \\
& \times\left[\gamma^{1 / 2} \psi+\sum_{k=0}^{(N-2) / 2} \operatorname{erf} \frac{x}{2 \sqrt{D_{\mathrm{ox}}} \sqrt{P+t^{\prime}}}\right. \\
& \left.-\sum_{k^{*}=1}^{(N-1) / 2} \operatorname{erf} \frac{x}{2 \sqrt{D_{\mathrm{ox}}} \sqrt{S+t^{\prime}}}\right]
\end{aligned}
$$


and

$$
\begin{aligned}
\frac{c_{\mathrm{re}}(x, t)}{c^{0}}= & \frac{\gamma^{1 / 2}}{1+\gamma^{1 / 2} \theta}\left[\sum_{k=1}^{(N+1) / 2} \operatorname{erf} \frac{x}{2 \sqrt{D_{\mathrm{re}}} \sqrt{A+t^{\prime}}}\right. \\
& \left.-\sum_{k=0}^{(N-2) / 2} \operatorname{erf} \frac{x}{2 \sqrt{D_{\mathrm{re}}} \sqrt{G+t^{\prime}}}\right] \\
& +\frac{\gamma^{1 / 2}}{1+\gamma^{1 / 2} \psi}\left[1+\sum_{k^{*}=1}^{(N-1) / 2} \operatorname{erf} \frac{x}{2 \sqrt{D_{\mathrm{re}}} \sqrt{S+t^{\prime}}}\right. \\
& -\sum_{k=0}^{(N-2) / 2} \operatorname{erf} \frac{x}{2 \sqrt{D_{\mathrm{re}}} \sqrt{P+t^{\prime}}}
\end{aligned}
$$

being $k^{\prime}=(k+1) / 2, k^{\prime \prime}=(k-2) / 2, k^{*}=(k-1) / 2$ where $1 \leq k \leq N$, and $\gamma=D_{\text {ox }} / D_{\text {re }}$

To simplify the expressions it has been considered that

$$
\begin{aligned}
& A=\left(k^{\prime}-1\right)\left(\tau_{\mathrm{a}}+\tau_{\mathrm{c}}\right), \quad B=\left(k^{\prime \prime}+1\right) \tau_{\mathrm{a}}+k^{\prime \prime} \tau_{\mathrm{c}}, \\
& E=k^{*} \tau_{\mathrm{a}}+\left(k^{*}-1\right) \tau_{\mathrm{c}}, \quad P=k^{\prime \prime}\left(\tau_{\mathrm{a}}+\tau_{\mathrm{c}}\right), \\
& G=\left(k^{\prime \prime}+1\right) \tau_{\mathrm{c}}+k^{\prime \prime} \tau_{\mathrm{a}}, \quad S=k^{*} \tau_{\mathrm{c}}+\left(k^{*}-1\right) \tau_{\mathrm{a}}
\end{aligned}
$$

From the concentration profiles of $c_{\mathrm{ox}}$ and $c_{\mathrm{re}}$ for odd and even values of $N$, equations (6) and (9), the fluxes of the reactants involved in the cathodic and anodic reactions expressed as current densities, $j_{c}$ and $J_{\mathrm{a}}$, respectively, can be immediately obtained For the cathodic current density

$$
\begin{aligned}
\mathrm{c}_{\mathrm{c}}= & -z \mathrm{~F} D_{\mathrm{ox}}\left(\frac{\partial c_{\mathrm{ox}}(x, t)}{\partial x}\right)_{x=0} \\
= & -\frac{z \mathrm{~F} D_{\mathrm{ox}}^{1 / 2} c^{0}}{\sqrt{\pi}}\left[\frac { 1 } { 1 + \gamma ^ { 1 / 2 } \theta } \left(\sum_{k=1}^{(N+1) / 2} \frac{1}{\sqrt{A+t^{\prime}}}\right.\right. \\
& \left.-\sum_{k=0}^{(N-2) / 2} \frac{1}{\sqrt{B+t^{\prime}}}\right)+\frac{1}{1+\gamma^{1 / 2} \psi}\left(\sum_{k^{*}=1}^{(N-1) / 2} \frac{1}{\sqrt{E+t^{\prime}}}\right. \\
& \left.\left.-\sum_{k=0}^{(N-2) / 2} \frac{1}{\sqrt{P+t^{\prime}}}\right)\right]
\end{aligned}
$$

and for the anodic current density

$$
\begin{aligned}
y_{\mathrm{a}}= & z \mathrm{~F} D_{\mathrm{re}}\left(\frac{\partial c_{\mathrm{re}}(x, t)}{\partial x}\right)_{x=0} \\
= & \frac{z \mathrm{~F} D_{\mathrm{ox}}^{1 / 2} c^{2}}{\sqrt{\pi}}\left[\frac { 1 } { 1 + \gamma ^ { 1 / 2 } \theta } \left(\sum_{k=1}^{(N-1) / 2} \frac{1}{\sqrt{A+t^{\prime}}}\right.\right. \\
& \left.-\sum_{k=0}^{(N-2) / 2} \frac{1}{\sqrt{G+t^{\prime}}}\right)+\frac{1}{1+\gamma^{1 / 2} \psi}\left(\sum_{k^{*}=1}^{(N-1) / 2} \frac{1}{\sqrt{S+t^{\prime}}}\right. \\
& \left.\left.-\sum_{k=0}^{(N-2) / 2} \frac{1}{\sqrt{P+t^{\prime}}}\right)\right]
\end{aligned}
$$

On the other hand, the cathodic and anodic charge densities, $q_{\mathrm{c}}$ and $q_{\mathrm{a}}$, comprised in each halfcycle, result from the integration of equations (10) and (11) respect- 1vely Thus, it results

$$
\begin{aligned}
q_{\mathrm{c}}= & \int_{0}^{\tau_{c}} \jmath_{\mathrm{c}} \mathrm{d} t \\
= & -\frac{z \mathrm{~F} D_{\mathrm{ox}}^{1 / 2} c^{0}}{\sqrt{\pi}}\left[\frac { 2 } { 1 + \gamma ^ { 1 / 2 } \theta } \left(\sum_{k=1}^{(N+1) / 2} \sqrt{H}\right.\right. \\
& \left.-\sum_{k=1}^{(N+1) / 2} \sqrt{A}+\sum_{k=0}^{(N-2) / 2} \sqrt{B}-\sum_{k=0}^{(N-2) / 2} \sqrt{L}\right) \\
& +\frac{2}{1+\gamma^{1 / 2} \psi}\left(\sum_{k=0}^{(N-2) / 2} \sqrt{P}-\sum_{k=0}^{(N-2) / 2} \sqrt{G}\right. \\
& \left.\left.+\sum_{k^{*}=1}^{(N-1) / 2} \sqrt{M}-\sum_{k^{*}=1}^{(N-1) / 2} \sqrt{E}\right)\right],
\end{aligned}
$$

and

$$
\begin{aligned}
q_{\mathrm{a}}= & \int_{0}^{\tau_{\mathrm{a}}} j_{\mathrm{a}} \mathrm{d} t \\
= & \frac{z \mathrm{~F} D_{0 \mathrm{x}}^{1 / 2} c^{0}}{\sqrt{\pi}}\left[\frac { 2 } { 1 + \gamma ^ { 1 / 2 } \theta } \left(\sum_{k=1}^{(N+1) / 2} \sqrt{U}\right.\right. \\
& \left.-\sum_{k=1}^{(N+1) / 2} \sqrt{A}+\sum_{k=0}^{(N-2) / 2} \sqrt{G}-\sum_{k=0}^{(N-2) / 2} \sqrt{L}\right) \\
& +\frac{2}{1+\gamma^{1 / 2} \psi}\left(\sum_{k=0}^{(N-2) / 2} \sqrt{P}-\sum_{k=0}^{(N-2) / 2} \sqrt{B}\right. \\
& \left.\left.+\sum_{k^{*}=1}^{(N-1) / 2} \sqrt{M}-\sum_{k^{*}=1}^{(N-1) / 2} \sqrt{S}\right)\right],
\end{aligned}
$$

where $H=k^{\prime} \tau_{\mathrm{c}}+\left(k^{\prime}-1\right) \tau_{\mathrm{a}}, L=\left(k^{\prime \prime}+1\right)\left(\tau_{\mathrm{a}}+\tau_{\mathrm{c}}\right), \quad M=$ $k^{*}\left(\tau_{\mathrm{a}}+\tau_{\mathrm{c}}\right), U=k^{\prime} \tau_{\mathrm{a}}+\left(k^{\prime}-1\right) \tau_{\mathrm{c}}$

22 The case of time-symmetric perturbation $\left(\tau_{\mathrm{c}}=\tau_{\mathrm{a}}\right)$

Let us consider the particular situation when $\tau_{\mathrm{c}}=$ $\tau_{\mathrm{a}}=\tau$, $i e$ a time-symmetric perturbing potential is applied to the electrode In this case from the generalized concentration profiles given by equations (6) to (9) one obtains for odd values of $N$

$$
\begin{aligned}
& \frac{c_{\mathrm{ox}}(x, t)}{c^{0}}=\frac{1}{1+\gamma^{1 / 2} \theta}\left[\gamma^{1 / 2} \theta+\sum_{k=1}^{N}(-1)^{k+1}\right. \\
& \left.\operatorname{erf} \frac{x}{2 \sqrt{D_{\mathrm{ox}}} \sqrt{(k-1) \tau+t^{\prime}}}\right]+\frac{1}{1+\gamma^{1 / 2} \psi} \\
& {\left[\sum_{k=2}^{N}(-1)^{k+1} \operatorname{erf} \frac{x}{2 \sqrt{D_{\mathrm{ox}}} \sqrt{(k-2) \tau+t^{\prime}}}\right],} \\
& \frac{c_{\mathrm{re}}(x, t)}{c^{0}}=\frac{\gamma^{1 / 2}}{1+\gamma^{1 / 2} \theta}\left[1+\sum_{k=1}^{N}\right. \\
& \left.(-1)^{k} \operatorname{erf} \frac{x}{2 \sqrt{D_{\mathrm{re}}} \sqrt{(k-1) \tau+t^{\prime}}}\right] \\
& +\frac{\gamma^{1 / 2}}{1+\gamma^{1 / 2} \psi}\left[\sum_{k=2}^{N}(-1)^{k} \operatorname{erf} \frac{x}{2 \sqrt{D_{\mathrm{re}}} \sqrt{(k-2) \tau+t^{\prime}}}\right]
\end{aligned}
$$


and for even values of $N$

$$
\begin{aligned}
& \frac{c_{\mathrm{ox}}(x, t)}{c^{0}}= \\
& \frac{1}{1+\gamma^{1 / 2} \theta}\left[\sum_{k=1}^{N}(-1)^{k} \operatorname{erf} \frac{x}{2 \sqrt{D_{\mathrm{ox}}} \sqrt{(k-1) \tau+t^{\prime}}}\right] \\
& +\frac{1}{1+\gamma^{1 / 2} \psi}\left[\gamma^{1 / 2} \psi+\sum_{k=2}^{N}(-1)^{k}\right. \\
& \left.\operatorname{erf} \frac{x}{2 \sqrt{D_{\mathrm{ox}}} \sqrt{(k-2) \tau+t^{\prime}}}\right] \\
& \frac{c_{\mathrm{re}}(x, t)}{c^{0}}=\frac{\gamma^{1 / 2}}{1+\gamma^{1 / 2} \theta}\left[\sum_{k=1}^{N}(-1)^{k+1}\right. \\
& \left.\operatorname{erf} \frac{x}{2 \sqrt{D_{\mathrm{re}}} \sqrt{(k-1) \tau+t^{\prime}}}\right]+\frac{\gamma^{1 / 2}}{1+\gamma^{1 / 2} \psi} \\
& {\left[1+\sum_{k=2}^{N}(-1)^{k+1} \operatorname{erf} \frac{x}{2 \sqrt{D_{\mathrm{re}}} \sqrt{(k-2) \tau+t^{\prime}}}\right]}
\end{aligned}
$$

From equatıons (14) and (17) results

$$
\begin{aligned}
& J_{\mathrm{a}, \mathrm{c}}= \\
& \quad \pm \frac{z \mathrm{~F} c^{0} D_{\mathrm{ox}}^{1 / 2}}{\sqrt{\pi}}\left[\frac{1}{1+\gamma^{1 / 2} \theta} \sum_{k=1}^{N}(-1)^{k+1}\right. \\
& \quad \frac{1}{\sqrt{(k-1) \tau+t^{\prime}}} \\
& \left.+\frac{1}{1+\gamma^{1 / 2} \psi} \sum_{k=2}^{N}(-1)^{k+1} \frac{1}{\sqrt{(k-2) \tau+t^{\prime}}}\right]
\end{aligned}
$$

and by integration to cover the time range $\tau$, the charge density equation is accomplished

$$
\begin{aligned}
& q_{\mathrm{ac}}= \pm \frac{z \mathrm{~F} D_{\mathrm{ox}}^{1 / 2} c^{0}}{\sqrt{\pi}}\left[\frac{4}{1+\gamma^{1 / 2} \theta}\right. \\
& \left((-1)^{k+1} \sum_{k=1}^{N-1} \sqrt{k \tau}+\frac{(-1)^{k+1}}{2} \sqrt{k \tau}\right) \\
& +\frac{4}{1+\gamma^{1 / 2} \psi}\left((-1)^{k+1} \sum_{k=1}^{N-2} \sqrt{k \tau}\right. \\
& \left.\left.+\frac{(-1)^{k-1}}{2} \sqrt{(k-1) \tau}\right)\right]
\end{aligned}
$$

The analysts of these equations and the corresponding experımental test are given in the following sections

\section{CONCLUSIONS FROM THE THEORETICAL EQUATIONS}

\section{The case $\tau_{\mathrm{c}}>\tau_{\mathrm{a}}$}

The equations determining the concentration profiles for ox and re can be plotted in a dimensionless form as $c_{\mathrm{ox}}(x, t) / c^{0}$ and $c_{\mathrm{re}}(x, t) / c^{0}$ vs $X^{*}$, where
$X^{*}=10 X / X_{\max }$ and $X_{\max }=4 \sqrt{D_{o x} \tau_{c}}$ This type of plot is depicted in Fig 1 for $\tau_{\mathrm{c}}=10^{-1} \mathrm{~s}, \tau_{\mathrm{a}}=10^{-3} \mathrm{~s}$ and $\Delta E=\left|E_{\mathrm{c}}-E_{\mathrm{r}}^{0}\right|=\left|E_{\mathrm{a}}-E_{\mathrm{r}}^{0}\right|=02 \mathrm{~V}$

The concentration profiles of ox and re which are bult up during the first cathodic halfcycle, $e N=1$ and $\tau_{c}$ (Fig 1a), are similar to those earlier described in the literature [7] for reaction (1) under a potential step In the first halfcycle, as the time of application of the potential perturbation increases, the thickness of the diffusion boundary layer also increases for both ox and re But a more complex situation arises for the subsequent halfcycles Hence, for each cathodic halfcycle, and a present value of $X^{*}$, the concentration of ox decreases as $t^{\prime}$ increases The reverse effect is noticed for the concentration of re Furthermore, for even values of $N, v$ anodic halfcycles, (Figs $1 \mathrm{~b}$ and $\mathrm{d}$ ) both concentration profiles exhibit for a certain value of $X^{*}$ a change in the sign of the derivative, corresponding to a minimum value of $c_{\mathrm{ox}}(x, t) / c^{0}$ and to a maximum value of $c_{\mathrm{re}}(x, t) / c^{0}$ For both species the bulk concentration is asymptotically attained as $X^{*}$ increases The values of $X^{*}$ where the minimum value of the dimensionless concentration of ox, and the maximum value of the dimensionless concentration of re are found, increase according to the value of $t^{\prime}$ for each halfcycle

Nevertheless, as the number of reduction-oxidation halfcycles increased the differences in the concentration profiles for different values of $t^{\prime}$ are progressively smaller, finally attaining concentration profiles which exhibit only modifications for small values of $X^{*}$ (Figs 1c and d)

Otherwise, when $\tau_{\mathrm{c}} / \tau_{\mathrm{a}} \gg 1$, as far as the development of the concentration profiles is concerned, the influence of the anodic halfcycle on the subsequent cathodic halfcycle becomes negligible, and the development of the ox concentration profile occurs nearly as if it is due exclusively to the cathodic step applied during the time $t$ In contrast, the influence of the cathodic halfcycle on the development of both the concentration profiles of re and ox during the subsequent anodic halfcycle is quite noticeable In the anodic halfcycles one can observe changes in the concentration profiles already for small values of $X^{*}$ which correspond to an increase in $c_{\mathrm{ox}}$ and a decrease in $c_{\mathrm{re}}$ as $N$ increases From $X^{*}=2$ upwards the concentration profiles tend to coincide with those resulting for the cathodic halfcycles

\section{The case $\tau_{\mathrm{a}}>\tau_{\mathrm{c}}$}

When the square wave perturbing potential involves the condition $\tau_{\mathrm{a}} / \tau_{\mathrm{c}} \geqslant 100$, the concentration profiles resulting for re and ox during the cathodic halfcycle are similar to those already known for reaction (1) proceeding under a constant potential step, $i e$ the concentration profile of ox increases monotonously with $X^{*}$ to approach the bulk solution concentration, whereas the opposite trend results for the concentration profile of re For both cases the diffusion layer becomes thicker as $t^{\prime}$ increases Furthermore, only a slight change in the concentration profiles with $N$ can be noticed

On the other hand, for the anodic halfcycles the changes of the concentrations of ox and re with $X^{*}$ and $t^{\prime}$ as well as with $N$ are very small 


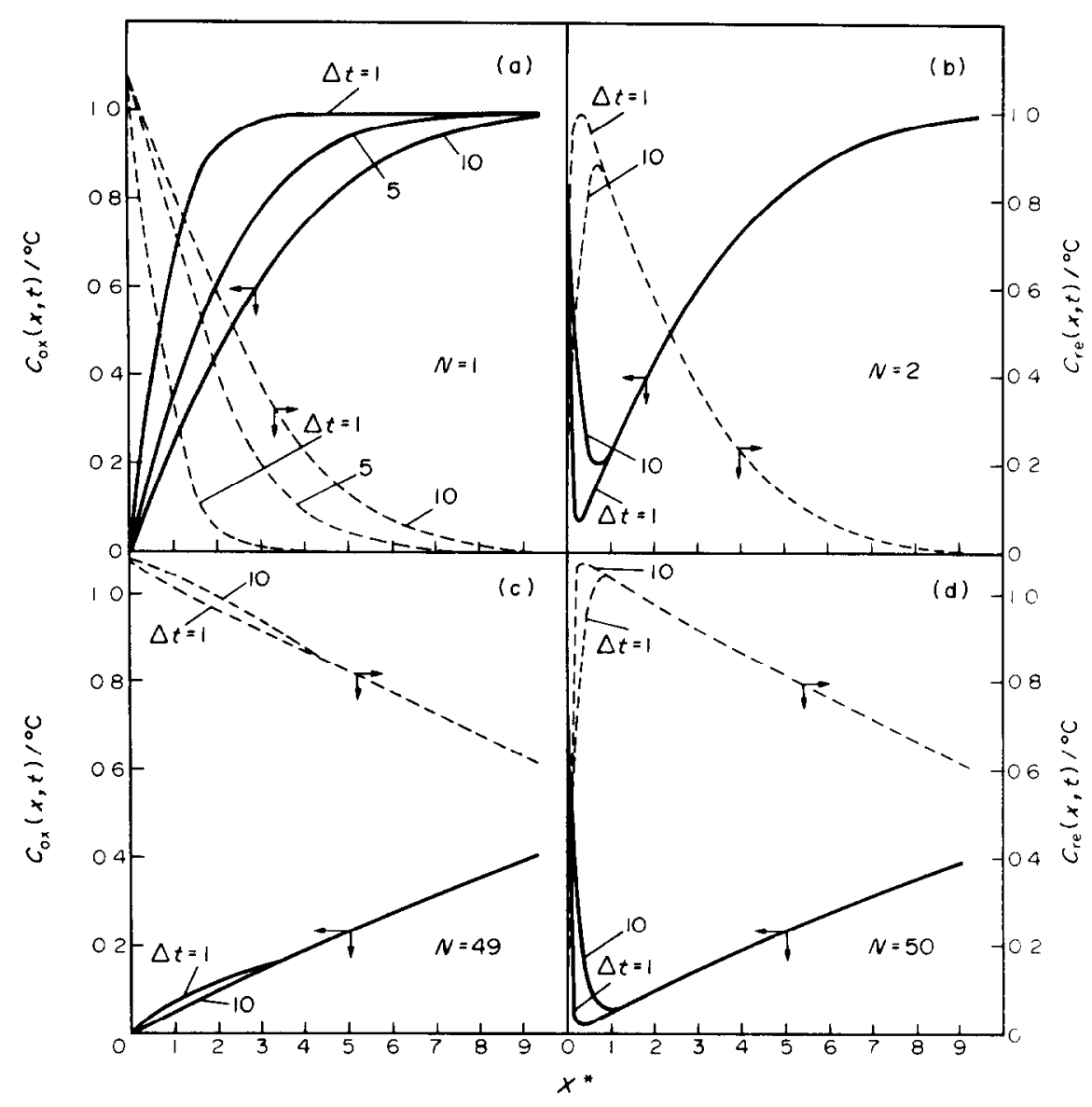

Fig 1 Concentration profiles for ox and re, resulting from the theoretical equation for a time-asymmetric square wave perturbing potential $\tau_{\mathrm{a}}=10^{-3} \mathrm{~s}, \tau_{\mathrm{c}}=01 \mathrm{~s}, D_{\mathrm{ox}}=6 \times 10^{-6} \mathrm{~cm}^{2} \mathrm{~s}^{-1}, D_{\mathrm{re}}=5 \times 10^{-6} \mathrm{~cm}^{2} \mathrm{~s}^{-1}$, $\Delta E=02 \mathrm{~V}$ (a) $1^{\text {st }}$ halfcycle (cathodic), $\Delta t=10 t^{\prime} / \tau_{\mathrm{c}}$, (b) $2^{\text {nd }}$ halfcycle (anodic), $\Delta t=10 t^{t} / \tau_{\mathrm{a}}$, (c) $49^{\text {th }}$ halfcycle (cathodıc), $\Delta t=10 t^{\prime} / \tau_{\mathrm{c}}$, (d) $50^{\text {th }}$ halfcycle (anodic), $\Delta t=10 t^{\prime} / \tau_{\mathrm{a}}$

\section{The symmetric case $\tau_{\mathrm{a}}=\tau_{\mathrm{c}}=\tau$}

Comparable conclusions can be derived for reaction (1) proceeding under a symmetric square wave perturbing potential The concentration profiles in this case are plotted by considering $\tau=1 \mathrm{~s}$ and $\left|E_{\mathrm{c}}-E_{\mathrm{r}}^{0}\right|$ $=\left|E_{\mathrm{a}}-E_{\mathrm{r}}^{0}\right|=02 \mathrm{~V}\left(\mathrm{~F}_{1} \mathrm{~g} 2\right)$ Again a conventional behaviour results for the first cathodic halfcycle $(N=1)$ (Fig 2a) Otherwise, for the first anodic halfcycle $(N=2)(\mathrm{F} i \mathrm{~g} 2 \mathrm{~b})$ as well as for other even values of $N$ (Fig 2d) one can observe a minımum value of $c_{\mathbf{o x}}$ for a certain value of $X^{*}$, and as $t^{\prime}$ increases the minimum value of $c_{\mathrm{ox}}$ moves towards greater values of $X^{*}$ and it tends to smear out Simultaneously, the value of the $c_{\mathrm{ox}} / c^{0}$ ratio increases according to $t^{\prime}$ As $X^{*}$ increases beyond the minimum value, the value of $c_{0 x}$ approaches asymptotically the concentration of ox in the bulk

The concentration profiles of ox and re resulting in the following cathodic halfcycles, $v e$ odd values of $N$, show a maximum value of $c_{\mathrm{ox}}$ and a minımum value of $c_{\text {re }}$ for small values of $t^{\prime}$ The values of $c_{\text {ox }}$ in the bulk of the solution decrease as $N$ increases In this case one can also observe that the maximal value of $c_{\text {ox }}$ and the mınımum value of $c_{\mathrm{re}}$ tend to disappear as $t^{\prime}$ increases
It should be noticed that for the symmetric perturbing potential the evolution of the concentration profiles for each halfcycle is largely influenced by the changes produced in the precedent halfcycle

On the other hand, the concentration profiles for $c_{\mathrm{re}}$ are nearly the specular Image of those already described for $c_{o x}$ (Figs $2 a-d$ )

\section{EXPERIMENTAL TEST OF THE THEORETICAL CONCLUSIONS}

The conclusions from the theory were verified with data obtained for the following electrochemical reaction

$$
\left[\mathrm{Fe}(\mathrm{CN})_{6}\right]^{3-}+\mathrm{e}^{-}=\left[\mathrm{Fe}(\mathrm{CN})_{6}\right]^{4-} \text {, }
$$

proceeding on a mirror polıshed platınum electrode under a square wave perturbing potential in the presence of a supporting electrolyte For the purpose of the present work reaction (20) on $\mathrm{Pt}$ can be considered as a sufficiently fast, reproducible and clean process under diffusion control 


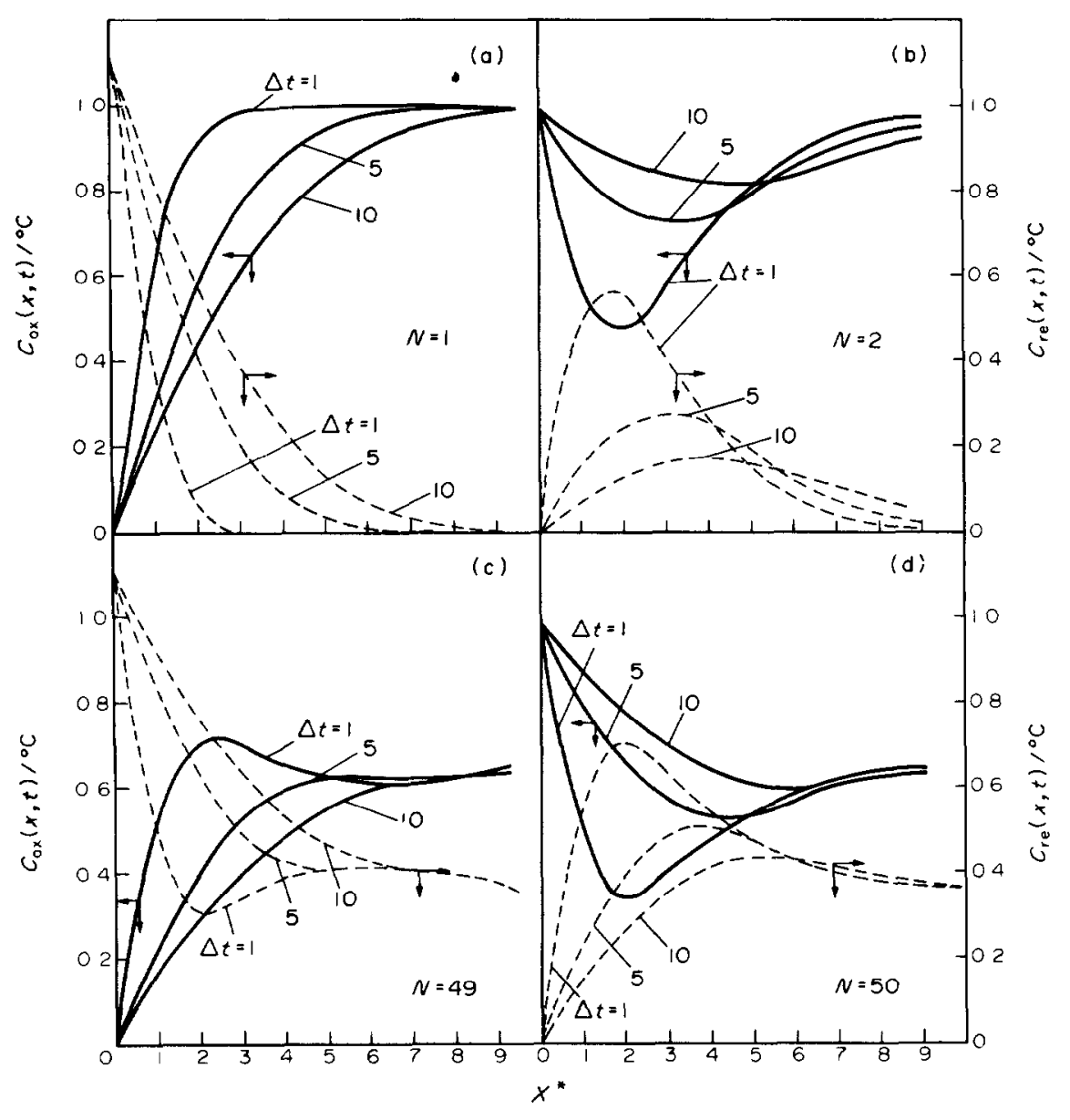

Fig 2 Concentration profiles for ox and re, resultıng from the theoretical equation for a time-symmetric square wave perturbing potentral $\tau=1 \mathrm{~s}, D_{\mathrm{ox}}=6 \times 10^{-6} \mathrm{~cm}^{2} \mathrm{~s}^{-1}, D_{\mathrm{re}}=5 \times 10^{-6} \mathrm{~cm}^{2} \mathrm{~s}^{-1}, \Delta E=02 \mathrm{~V}$, $\Delta t=10 t^{\prime} / \tau$ (a) $1^{\text {st }}$ halfcycle (cathodic), (b) $2^{\text {nd }}$ halfcycle (anodic), (c) $49^{\text {th }}$ halfcycle (cathodic), (d) $50^{\text {th }}$ halfcycle (anodic)

\section{Experimental}

The electrochemical runs were performed in a single compartment Pyrex glass cell of about $200 \mathrm{~cm}^{3}$ capacity The working electrode consisted of a $\mathrm{Pt}$ sheet of $0096 \mathrm{~cm}^{2}$ geometric area mounted into a glass picce The counter electrode was also made from a $\mathrm{Pt}$ sheet, its geometric area was $4 \mathrm{~cm}^{2}$, and it was arranged parallel with respect to the working electrode a distance sufficiently shorter to achieve a primary current distribution as homogeneous as possible A saturated calomel electrode (sce) connected through a capillary tip close to the working electrode and properly shielded to avoid $\mathrm{Hg}_{2}^{2+}$ ion diffusion into the cell was employed as reference The electrolyte solution was prepared from $\mathrm{K}_{3}\left[\mathrm{Fe}(\mathrm{CN})_{6}\right]$, Merck $\mathrm{pa}, \mathrm{NaOH}$, Carlo Erba $p a$, and MillQ water, and the initial concentration of the reacting species, $c^{0}$, was determined through conventional chemical analysis The solution composition used throughout the work was $001 \mathrm{M} \mathrm{K}_{3}\left[\mathrm{Fe}(\mathrm{CN})_{6}\right]+1 \mathrm{M} \mathrm{NaOH}$ Runs were made at $25^{\circ} \mathrm{C}$

The square wave perturbıng potentıal was applied through a fast rise tıme potentiostat operated by a square wave generator The current response was recorded through a Nicolet oscilloscope The frequency range of the perturbing potential was $10^{4} \mathrm{~Hz} \geqslant f \geqslant 5 \times 10^{-2} \mathrm{~Hz}$ and the value of $\Delta E$ was $\pm 02 \mathrm{~V}$

\section{Comparison of experimental results and theoretical predictlons}

421 Current transients Let us first consider that $\tau_{\mathrm{c}}=01 \mathrm{~s}, \tau_{\mathrm{a}}=10^{-3} \mathrm{~s}$, ie $\tau_{\mathrm{a}}<\tau_{\mathrm{c}}$, and $\left|E_{\mathrm{c}}-E_{\mathrm{c}}^{\mathrm{o}}\right|=\mid$ $E_{\mathrm{a}}-E_{\mathrm{r}}^{0} \mid=02 \mathrm{~V}$ The comparison of the theoretical and experimental data, as shown in Fig 3, is certainly very good The current response for the successive cathodic halfcycles generates an envelope which tends to coincide with that expected for a single potential step with the value of $\Delta E$ applied during the time $t$ The cathodic current decreases along the subsequent cathodic halfcycles

On the other hand when $\tau_{\mathrm{a}}>\tau_{\mathrm{c}}$, again a good coincide between theory and experiment is obtained In this case one observes a rapid trend of the cathodic current transient to reach a stationary reproducible 


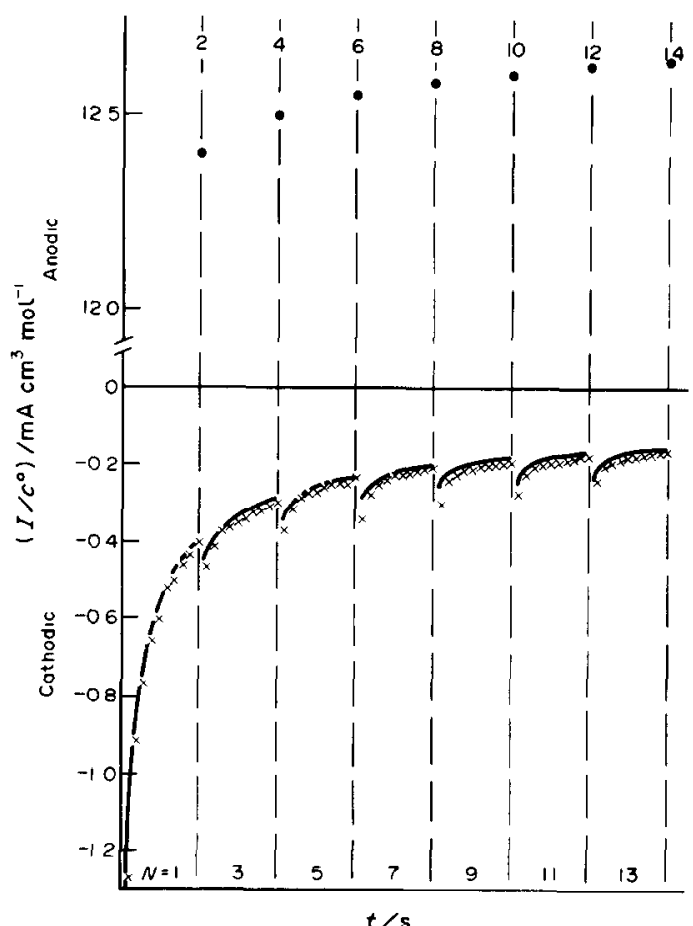

Fig 3 Comparison between theoretical and expcrimental current transients $v s$ the number of halfcycles for a tumeasymmetric square wave perturbing potential $\tau_{\mathrm{a}}=10^{-3} \mathrm{~s}$, $\tau_{\mathrm{c}}=01 \mathrm{~s}, \Delta E=02 \mathrm{~V}$

situation, which can be related to the fact that the low anodic current values reached, te $50-100 \mu \mathrm{A}$, becomes sufficient to allow the complete disappearance of the small amount of re produced in the preceding cathodic halfcycle

Finally, it is interesting to analyze data for the symmetric case, $\tau_{\mathrm{c}}=\tau_{\mathrm{a}}=\tau$ In this case, both anodic and cathodic current transients obtained for $\tau=1 \mathrm{~s}$ (Fig 4) decay relatively rapid according to the duration of the halfcycle Nevertheless, in any case, it appears that for a fixed value of $t^{\prime}$ the current in the anodic halfcycles is always rather smaller than in the cathodic halfcycles

On the other hand, the current in the anodic halfcycles read for $t^{\prime}=\tau$ tends to increase as $N$ increases, as it should occur according to the concentration profiles (Fig 2) when the concentration of re in solution has been increased Similarly, the current for the cathodic halfcycles diminishes as $N$ increases, as it should be expected for a gradual decrease in ox concentration with $t^{\prime}$ Once more the agreement between theory and experiment is quite satisfactory Similar results have been also obtained for the three cases in the enture frequency range covered in the present work

422 Charge density plots From equations (12) and (13) it is possible to calculate the amount of product going into solution along the potential cycling This calculation for the case of the asymmetric square wave perturbing potentials is shown in Fig 5 In this case one can observe that for $\tau_{\mathrm{a}}=10^{-3} \mathrm{~s}$,

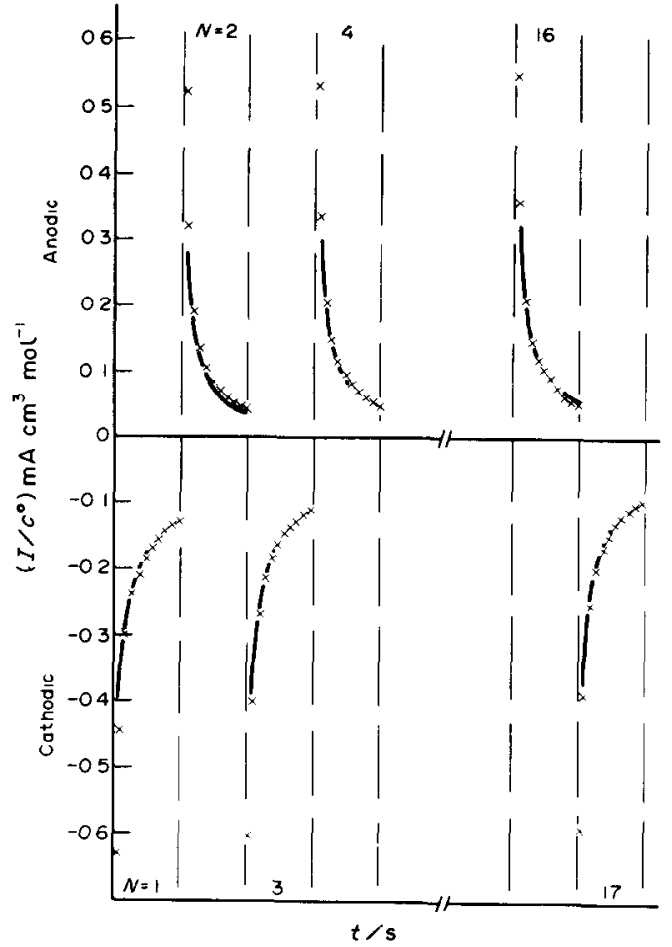

Fig 4 Comparison between theoretical and experımenta] current transient $v s$ the number of halfcycles for a tımesymmetric square wave perturbing potentıal $\tau=1 \mathrm{~s}$, $\Delta E=02 \mathrm{~V}$

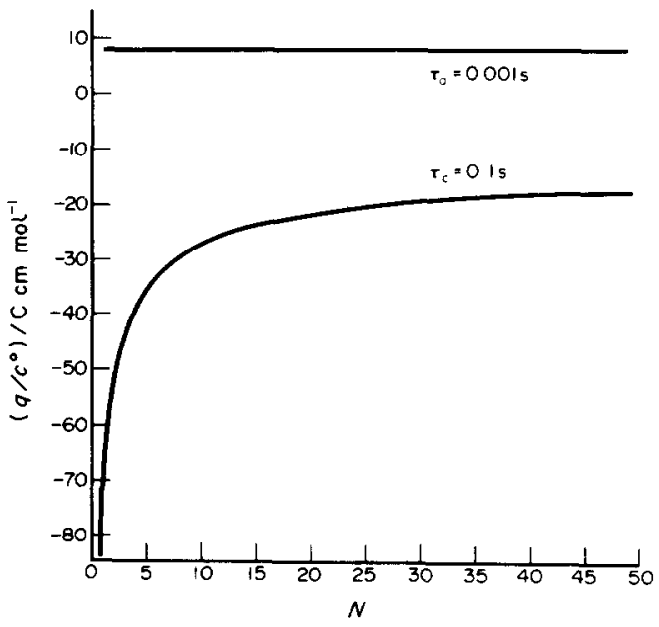

Fig 5 Normalized charge densitıes $\left(q / c^{\circ}\right)$ for anodic and cathodic halfcycles $i s$ the number of halfcycles, for a timeasymmetric square wave perturbing potentral $\tau_{\mathrm{a}}=10^{-3} \mathrm{~s}$,

$$
\tau_{\mathrm{c}}=01 \mathrm{~s}, \Delta E=02 \mathrm{~V}
$$

$\tau_{\mathrm{c}}=01 \mathrm{~s}$ and $\Delta E=02 \mathrm{~V}$, the cathodic charge decreases sharply with $N$, whereas the anodic charge remains practically constant

The charge difference, $\Delta q$, accumulated along the successive oxidation-reduction cycles can be immedi- 
ately calculated through the following relationship

$$
\Delta q=\sum_{k=0}^{N} q_{\mathrm{a}}\left(k^{\prime \prime}\right)-\left|\sum_{k=1}^{N} q_{\mathrm{c}}\left(k^{\prime}\right)\right|
$$

A plot of $\Delta q v s N$ is illustrated in Fig 6 by using for this case the values of $\tau_{\mathrm{a}}, \tau_{\mathrm{c}}$ and $\Delta E$ referred to above This plot shows an increase in the cathodic charge, $v e$ an increase of the concentration of re in solution, as $N$ is increased Certainly, the value of $\Delta q$ depends on the number of halfcycles considered and on the frequency of the periodic perturbation

On the other hand, when the same analysis is extended to the symmetric case, $\imath e \tau_{\mathrm{a}}=\tau_{\mathrm{c}}=\tau$, for $\tau=1 \mathrm{~s}$ and $\Delta E=02 \mathrm{~V}\left(\mathrm{~F}_{1 \mathrm{~g}} 7\right)$, the charge accumulated per anodic halfcycle increases according to $N$, but, the opposite effect is observed for the cathodic halfcycle For the latter the change of $q$ with $N$ for the symmetric case is much smoother than that already described for the asymmetric one The $\Delta q$ vs $N$ plots for the symmetric case (Fig 8) show qualitatıvely the same behaviour already described for the $\Delta q v s N$ plots for the asymmetric case

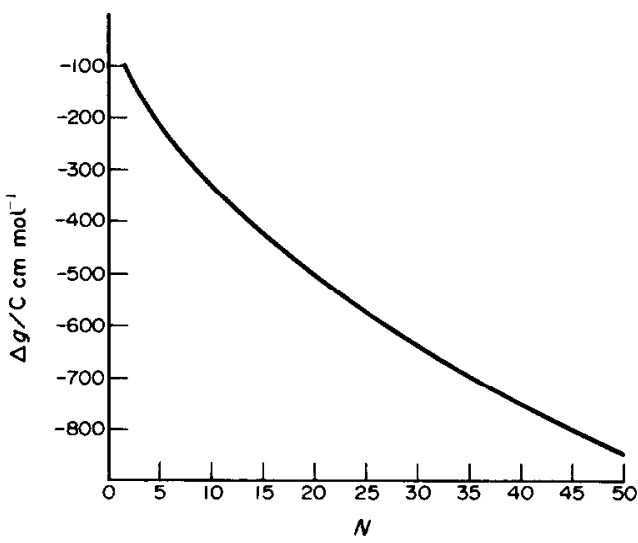

Fig 6 Plot of $\Delta q$ vs the number of halfcycles, for a timeasymmetric square wave perturbing potentıal $\tau_{\mathrm{a}}=10^{-3} \mathrm{~s}$,

$$
\tau_{\mathrm{s}}=01 \mathrm{~s}, \Delta E=02 \mathrm{~V}
$$

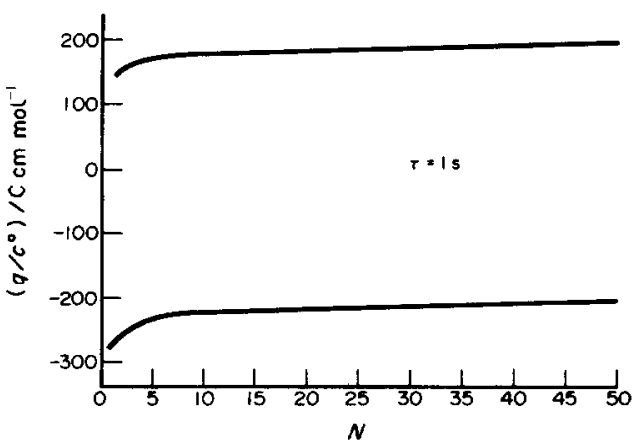

Fig 7 Normalized charge densities $\left(q / c^{\circ}\right)$ for anodic and cathodic halfcycles vs the number of halfcycles, for a timesymmetric square wave perturbıng potential $\tau=1 \mathrm{~s}$, $\Delta E=02 \mathrm{~V}$

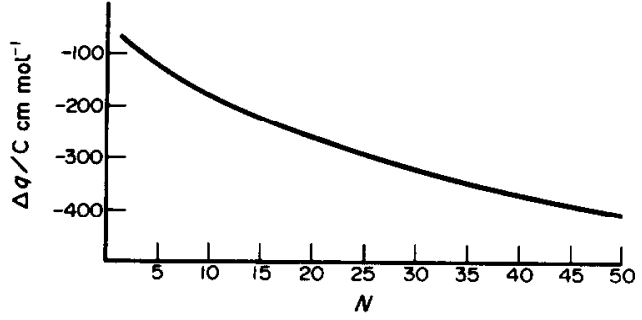

Fig 8 Plot of $\Delta q$ vs the number of halfcycles for a tumesymmetric square wave perturbing potential $\tau=1 \mathrm{~s}$, $\Delta E=02 \mathrm{~V}$

\section{CONCLUSIONS}

The model allows to calculate the concentration profiles at any tıme of reactants and products for a diffusion controlled electrochemical redox reaction involving soluble species in solution under a square wave perturbing potential where only the reactant is present at the initiation of the reaction

The equations for the charge density and current density transients at each halfcycle are also obtained from the model The influence of the applied potential, duration $\left(t^{\prime}\right)$ and halfperiod on the current can be evaluated A linear relationship between the current density and the reactant concentration originally present in the solution has been established

When $\tau_{\mathrm{a}} / \tau_{\mathrm{c}}=1$, the current transients exhibit no substantial variation with the number of halfcycles The absolute values of the current for the anodic and cathodic halfcycles are of the same order of magnitude, despite the fact that for a constant $t^{\prime}$ value the absolute values of the current in the anodic halfcycles increase and in the cathodic halfcycles decrease according to $N$

Otherwise, when $\tau_{\mathrm{a}} / \tau_{\mathrm{c}}<1$ the cathodic current decreases with $N$, and it is possible to draw a current time envelope including all halfcycles which approaches very closely the current transient predicted for a constant single potential step applied to reaction model during a time equal to the sum of $\tau_{c}$ for the cathodic halfcycles The better the approach the lower the $\tau_{\mathbf{a}} / \tau_{\mathbf{c}}$ ratio

Finally, when $\tau_{\mathrm{a}} / \tau_{\mathrm{c}}>1$, a fast stabilization of the current transients is observed for both anodic and cathodic halfcycles Nevertheless, it should be noticed that for $\tau_{a} / \tau_{c}>10^{3}$ negative currents in the anodic halfcycles can be noticed The equations developed from the model indicate that throughout the application of square wave perturbing potential it is possible to change at will the relative rates of the anodic and cathodic reactions

Acknowledgement-This research project was financially supported by the Consejo Nacional de Investigaciones Cientıficas y Tecnicas and the Comision de Investigaciones Cientificas de la Provincia de Buenos Aires

\section{REFERENCES}

1 J C Canullo, W E Triaca and A J Arvia, $J$ electroanal Chem 175, 337 (1984) 
2 A Visintin, J C Canullo, W E Triaca and A J Arvia, $J$ electroanal Chem 239, 67 (1988)

3 E Yedger and A J Salkınd, in Techniques of Electrochemistry, $J$ Wiley, New York (1973)

4 A R Despic and K I Popov, J appl Electrochem 1, 275 (1971)

5 S L Marchiano, L Rebollo Neıra and A J Arvia, Electrochim Acta, in press

$6 \mathrm{~S} \mathrm{~L}$ Marchiano and $\mathrm{A} \mathrm{J}$ Arvia, in Comprehensive Treatise of Electrochemistry (Edited by E Yeager, J O'M
Bockris, B E Conway and S Sarangapan1), Ch 2, p 65, Plenum Press, New York (1983)

7 P Delahay, New Instrumental Methods in Electrochemtstry, Interscience, New York, (1966)

8 C I Elsner, S L Marchıano and A J Arvıa, in preparation

$9 \mathrm{~J}$ Newman, in Advances in Electrochemistry and Electrochemical Engineering (Edited by P Delahay and Ch W Tobias), Vol 5, p 87, Interscience, New York (1966) 